\title{
Without Clay Drilling Fluids for Well Bore Wiring in Complicated Conditions
}

\author{
Raupov A.A., Gaibnazarov S.B.
}

\begin{abstract}
The causes of collapse of clay rocks of different Genesis were revealed. As a result of the experimental study, a chalk solution stabilized with non-hydrolyzed polyacrylamide was proposed. The assessment of the degree of stability of clay cores, both freshwater and marine origin in the process of washing wells with chalk solution stabilized polymer reagents. In order to ensure the stability of the wellbore wall, it is recommended to open potentially unstable clay deposits using chalk solutions stabilized with non-hydrolyzed polyacrylamide.
\end{abstract}

Keywords : neogenic deposits, polyvalent cations, hydroloads, neogen, paleogen, clay, polyacrylamide (PAA), clay rocks, continental-freshwater conditions.

\section{INTRODUCTION}

The section of the majority of exploration and operational areas of our Republic is $60-70 \%$ represented by clay rocks of different genesis [1]. The clay material herein is represented mainly by a mixture of hydroslude and montmorillonite.

With the increase in the depth of the wells, i.e. with the transition from neogen to paleogen and further to Cretaceous sediments, there is a natural decrease in montmorillonite content in the rocks and an increase in the proportion of hydroloads. Neogenic deposits in interhore depressions (Fergana and Amudarya) were formed in continental-freshwater conditions, and Paleogenic and Cretaceous deposits were formed in marine conditions [2]. Therefore, paleogenic clays contain water-soluble pore salts, mainly sodium chloride, sodium and calcium sulfates. Neogenic deposits are "fresh," as clay rocks contain montmorillonite and hydrosprum, they are prone to swelling.

The swelling effect is due to both the ionic composition of the mud salts. When $\mathrm{Na}^{+}$serves as the inter-pack ion compensator, the clay is confirmed to swell by fresh drilling fluids. If the ions of compensators in the clay mineral are polyvalent cations $\mathrm{Ca}^{++}, \mathrm{Mg}^{++}$and $\mathrm{Al}^{+++}$, the swellability of the clays by fresh water is dramatically slowed. If salts containing polyvalent cations, such as $\mathrm{CaCl}_{2}$, are added to the solution, chemical and electro kinetic stability will be

Revised Manuscript Received on December 30, 2019.

* Correspondence Author

Raupov Anvar Abdirashidovich, Senior Researcher - Applicant, Tashkent State Technical University, Tashkent, Uzbekistan Email: raupov.a@gov.uz

Gaibnazarov Sunnatilla Bahodirjanovich*, Dean of the Department of Mining and Metallurgy, Tashkent State Technical University, Tashkent, Uzbekistan Email: sunnatilla77@mail.ru

(C) The Authors. Published by Blue Eyes Intelligence Engineering and Sciences Publication (BEIESP). This is an open access article under the CC BY-NC-ND license (http://creativecommons.org/licenses/by-nc-nd/4.0/) improved. By penetrating between the packets, increase the coupling energy between them even at low concentrations.

\section{OBJECTS AND METHODS RESEARCH}

At the same time, there is a significant compression of the double electric layer, hence the clay rock with respect to the $\mathrm{CaCl}_{2}$-containing drilling mud should have a sufficiently high stability already with small concentrations of this salt in the solution. It is therefore advantageous to use mineralized drilling fluids containing polyvalent cations to guide wells under continental clay conditions.

High mineralization solutions are necessary to eliminate or significantly reduce the process of formation of sea-derived clay rocks (paleogen, chalk and Senonic clays). Diffusion processes of pore salts removal into drilling fluid and osmotic phenomena are excluded or slowed down. As mineralizers of drilling mud it is expedient to use the salts containing polyvalent cations $\left(\mathrm{MgCl}_{2}\right.$ and $\left.\mathrm{CaCl}_{2}\right)$. In their application, attenuation of energy of connection between packets of clay particles and peptization are excluded. As is known, in the conditions of our Republic clay solutions are still used for mass drilling on a wide scale. Clay drilling fluids are known to be highly sensitive to changes in solution mineralization. This phenomenon is associated with aggregation of fine clay particles as a result of compression of the double electric layer while increasing mineralization. During well drilling as a result of ingress of formation water salts and rock salts into clay solutions, their coagulation thickening or liquefaction is caused, and multiple chemical treatment with expensive reagents was required to restore the solution properties. In addition, the use of clay solutions for well washing does not provide a high rate of well construction increases labor costs per meter of penetration, deep penetration of solid clay phase into pores of the productive horizon leads to irreversible colmatration of the formation. Therefore, we have proposed salt-resistant drilling fluids in which a cretaceous suspension serves as a dispersed phase to guide wells in sections represented by powerful clay deposits and to open productive beds of the ANPD. The advantage of this solution is that the density can be adjusted within wide limits without the addition of weighting agents. Chalk particles are practically free from spontaneous dispersal. The Cretaceous solution can be easily acid treated, which allows to completely restore natural filtration properties of productive formations after their initial opening by drilling. The stability of the Cretaceous solution is independent of its mineralization. However, the Cretaceous solution has a significant disadvantage - weak sedimentation resistance. In order to increase structural stability, we propose to introduce non-hydrolyzed polyacrylamide (PAA) into the solution composition. PAA, according to experiments 
Without Clay Drilling Fluids for Well Bore Wiring in Complicated Conditions

Table 1 : Technological characteristics of clay and cretaceous solutions with addition of chemical reagents

\begin{tabular}{|c|c|c|c|c|c|c|c|}
\hline \multirow[b]{2}{*}{ № } & \multirow[b]{2}{*}{ Composition of Solution } & \multirow[b]{2}{*}{ 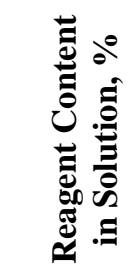 } & \multicolumn{5}{|c|}{ Indicators solution } \\
\hline & & & 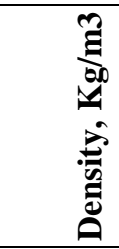 & 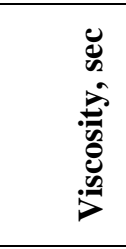 & 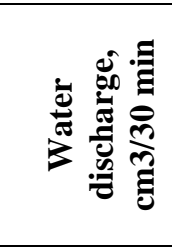 & 氖 & $\mathbf{p H}$ \\
\hline \multirow[t]{5}{*}{1} & Bentonite clay powder solution & - & 1100 & 57 & 23 & 5 & 7,0 \\
\hline & Solution with additive CMS-700 & 0,5 & 1105 & 44 & 9,0 & 3 & 8,0 \\
\hline & Solution with addition of carbo-PAC & 0,5 & 1107 & 50 & 7,0 & 3 & 8,0 \\
\hline & Solution with addition of uniforms & 0,5 & 1107 & 48 & 6,0 & 3 & 9,0 \\
\hline & Solution with added PAA & 0,1 & 1110 & - & $40 / 15$ & 6 & 9,0 \\
\hline \multirow[t]{5}{*}{2} & Solution from Nawbakhor clay powder & - & 1250 & 53 & 29,0 & 6 & 7,0 \\
\hline & Solution with additives CMS-700 & 0,5 & 1240 & 41 & 12,0 & 4 & 8,0 \\
\hline & Solution with addition of carbo-PAC & 0,5 & 1240 & 55 & 9,0 & 2 & 9,0 \\
\hline & Solution with addition of uniforms & 0,5 & 1240 & 45 & 7,0 & 3 & 9,0 \\
\hline & Solution with added PAA & 0,1 & 1245 & - & $40 / 15$ & 7 & 9,0 \\
\hline \multirow[t]{6}{*}{3} & Cretaceous solution & - & 1350 & 65 & $25 / 20$ & 8 & 8,0 \\
\hline & Solution with additive КМЦ-700 & 0,5 & 1340 & 55 & 15,0 & 6 & 8,0 \\
\hline & Solution with addition of carbo-PAC & 0,5 & 1340 & 47 & 8,0 & 4 & 8,0 \\
\hline & Solution with addition of uniforms & 0,5 & 1340 & 45 & 5,0 & 3 & 8,0 \\
\hline & Solution with added PAA & 0,1 & 1350 & 53 & 12 & 6 & 8,0 \\
\hline & Solution with added PAA & 0,5 & 1345 & 37 & 4,0 & 2 & 8,0 \\
\hline
\end{tabular}

time, the viscosity index reaches the required values

performed by us [3], has a surface positive charge. Therefore, when absorbed on the Cretaceous particles, the polyacrylamide imparts to the solution as a whole the properties of a colloidal system capable of forming a coagulation structure. Entering further coagulation, fine chalk particles coated with non-hydrolyzed polyacrylamide form a structural framework that slows down or prevents sedimentation processes. Simultaneously, the adsorbed PAA on the surface of the Cretaceous particles serves as a structural mechanical barrier preventing the Cretaceous particles from sticking together.

\section{EXPERIMENTS AND RESULTS}

For experimental confirmation of the proposed theoretical prerequisites, we have made special experiments. In the experiments, the main indicators of the proposed solution depending on the percentage of addition of PAA and chalk are considered. Characteristics of the solution on the basis of stabilized CMC clay powder, uniforms, carbo-PAC and PAA were considered as comparison standards. The main indicators against which drilling fluids were compared were viscosity, filtration, clay crust thickness and $\mathrm{pH}$. Table 1 shows the characteristics of clay and cretaceous solutions with stabilized chemicals.

As can be seen from these tables, the reduction of $\mathrm{PAH}$ $0.1 \%$ into clay solutions prepared from both bentonite and Navbahar clay powder dramatically impairs its properties, in particular thickening and increasing filtration, which is caused by aggregation of clay particles as a result of coagulation processes. At the same time, the performance of

the $\mathrm{PAH}$ additive in the Cretaceous solution is significantly improved. Thus, if the filtration of the Cretaceous solution without additives was $25 \mathrm{~cm}^{3}$ in 20 minutes, it was reduced to $4 \mathrm{~cm}^{3}$ in 30 minutes when 0,5 PAA was introduced, the value of the crust was only $2,0 \mathrm{~mm}$. Compared to the thickness of the crust poured by the original Cretaceous solution, it decreased by a factor of 4 . At the same
Reference should also be made to the high sedimentation stability of the PAA-added Cretaceous solution. The DST of this solution is zero. As can be seen from the data in Table 1, the additives of the remaining chemical did not provide such high levels of Cretaceous solution of stabilized polyacrylamide. Only the addition of the uniform can compete with the PAA in terms of such indicators as filtration capacity and crust thickness. However, uniflok is a scarce and expensive chemical reagent.

To test the above hypothesis, we evaluated the degree of dispersion of both natural and artificially prepared clay cores.

As a criterion for assessing the degree of stability of clay rocks, the dispersing capacity of clays in aqueous solutions was adopted. In the experiments, salts of $\mathrm{NaCl}$ $\mathrm{CaCl}_{2}$ and $\mathrm{MgCl}_{2}$ 5\% concentration alone and a mixed increased dose of chlorides were used as drilling fluids. Figure 1 shows the nature of the dependence curves of the dispersion values of clays of continental freshwater origin in solutions of $\mathrm{NaCl} \mathrm{CaCl} 2$ and $\mathrm{MgCl}_{2} 25 \%$ concentration.

Analysis of the curves showed that the clay core dispersed completely in fresh water for 20 days. In $\mathrm{NaCl}$ solutions, the amount of dispersion of clay cores of the continental freshwater genesis has a maximum value than in $\mathrm{CaCl}_{2}$ and $\mathrm{MgCl}_{2}$ solutions. Apparently, this phenomenon indicates a process of restructuring the exchange complex of cations with high valence. At the same time energy of connection between packets decreases, intensity of dispersion and peptization increases. 


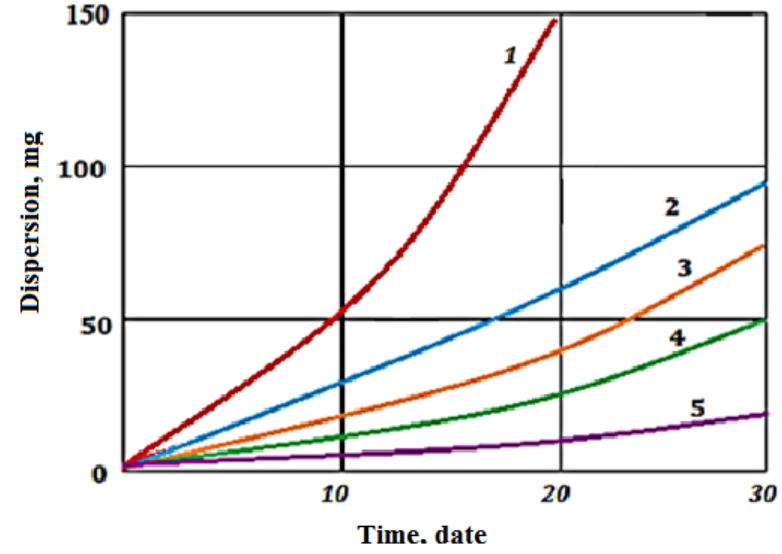

Fig. 1. Dependence of dispersion value of clays of continental-freshwater origin in solutions: 1 - fresh water; 2 - 5\% $\mathrm{NaCl}$ solution; 3 -5\% $\mathrm{CaCl}_{2}$ solution; 4 -5\% $\mathrm{MgCl}_{2}$ solution; 5 - mixed (5\%) 15\% chloride salt concentration

Mixed salt concentrations have minimal dispersion values. The clay core was dispersed for only $20 \mathrm{mg}$ for 30 days. The results of the experiments are fully consistent with the theoretical assumptions put forward by the competing influence of factors that contribute to the chemical and electro-kinetic stability of clay rocks.

If a complex of salts $\left(\mathrm{NaCl}, \mathrm{CaCl}_{2}\right.$ and $\left.\mathrm{MgCl}_{2}\right)$ is used as a mineralizer, such a pattern greatly increases the bond strength between the packages.

As noted, sea genesis clays are represented mainly by montmorillonite hydrates, and pore salts include $\mathrm{MgCl}_{2}$ $\mathrm{CaCl}_{2}$ and $\mathrm{NaCl}$.

In order to study the sea genesis clay rocks for the degree of stability, we produced artificial cores saturated with $\mathrm{MgCl}_{2} \mathrm{CaCl}_{2}$ and $\mathrm{NaCl} 5 \%$ separately and $15 \%$ mixed (5\% each salt).

Figure 2 shows the results of the dispersion of sea genesis clays in solenous solutions.

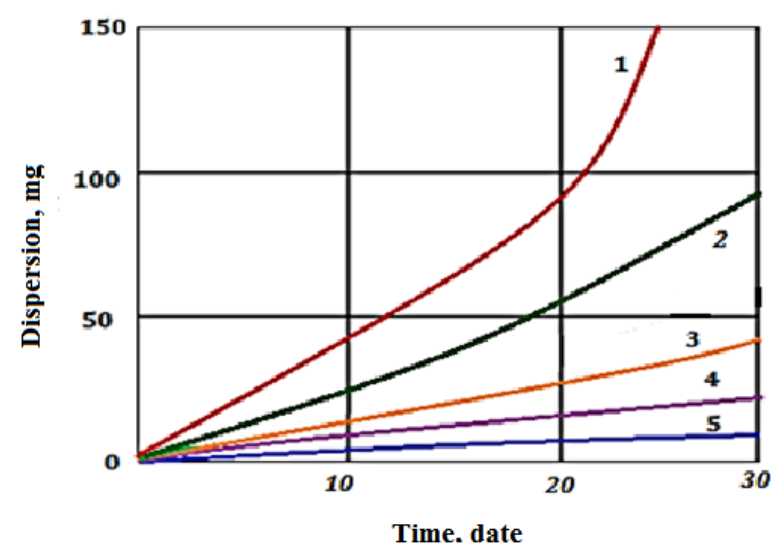

Fig. 2. Dispersion of sea genesis clays in solenous solution: 1 - fresh water; $2-5 \% \mathrm{NaCl}$ solution; $3-5 \% \mathrm{CaCl}_{2}$ solution; 4 -5\% $\mathrm{MgCl}_{2}$ solution; 5 - mixed (5\% each) 15\% solution of $\mathrm{CaCl}_{2}$ salts, $\mathrm{NaCl}$ and $\mathrm{MgCl}_{2}$

As can be seen, the dispersal effect is dramatically reduced in all mineralized solutions compared to fresh water. It is interesting to note that the lowest dispersion is observed in clays, $\%$ of mixed salinous chloride solutions. This once again indicates that sodium, calcium, and magnesium chlorides significantly increase the bond energy between the packages of hydrous and montmorillonite clays of marine genesis.
Compared to fresh water in $\mathrm{NaCl}$, and $\mathrm{CaCl}_{2}$ and $\mathrm{MgCl}_{2}$ solutions, clays significantly increase resistance, both continental and marine genesis. In [3], it was noted that PAA, by neutralizing the surfaces of the charge of clay particles, initially causes weak coagulation. However, with the expiration of time due to slow hydrolysis processes, the solution becomes stable by forming a double electric layer on the surface of the Cretaceous particles. In this regard, there is reason to suggest that the introduction of PAA into drilling mud should have a positive effect on the sustainability of clay rocks, both continental freshwater and marine genesis. In order to test the proposed hypothesis, we evaluated the degree of dispersion of clay cores in the Cretaceous solution, and with the addition of $0.5 \%$ PAA and gave a comparative assessment of the uniflok. For this purpose, drilling mud filtrates were prepared and sea clay cores were placed in them strongly and weakly salted as well as continental.

Figure 3 shows the dispersion curves of clays in a $0.5 \%$ PAA-added Cretaceous solution.

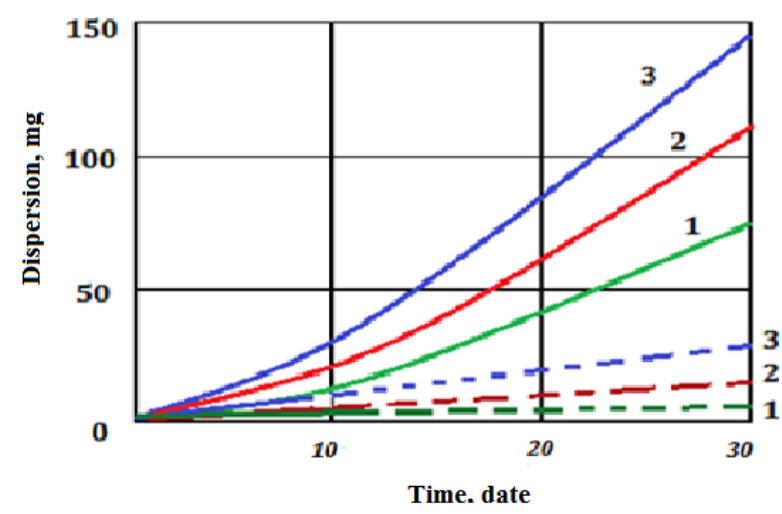

Fig. 3. Dispersion of clay cores in a Cretaceous solution: 1 - Jurassic clay - continental; 2 - senon clay slightly salted; 3 - paleogen clay - strongly ground; with uniflock -------, additive with PAA additive - -

\section{CONCLUSION}

As would be expected, sea genesis clay (paleogen) has the smallest Jurassic continental freshwater clay. When $0,5 \%$ of PAA was added within 15 days, the instrument recorded a not significant amount of core dispersion of all the clays tested. The study thus suggests that the developed PAA-added Cretaceous drilling mud has a high structural stability over a wide range of changes in its mineralization. When conducting wells under conditions of clay rocks of different genesis, it is possible to reduce the probability of swelling and roll formation of rocks forming walls of well bore.

\section{REFERENCES}

1. A.M. Aminov. Drilling deep wells in complicated conditions. Tashkent, 1992 -C. 11.

2. Sh.M. Rahimbayev. On the use of facial-paleographic maps in the extension of wells. Drilling of oil and gas wells in complicated conditions. Tashkent, Works of SAIGMS, vol. 24, 1976. -C 63-65.

3. A.A. Raupov. Selection of non-hydrolyzed polyacrylamide (PAA) as an effective reagent for treating Cretaceous solutions. Moscow: Gas Industry, 2019.

4. Gaibnazarov S.B. Physicochemical and macromolecular characteristics of new stabilizers of drilling fluids//Chemistry and chemical technology. 2017. №1 (55). Page 48-52. 
5. Gaibnazarov S.B. Study of influence of polymer reagents on thixotropy of drilling fluids//Chemical industry. 2016. Vol. 93 No. 5. Page 258-261.

6. Gaibnazarov S.B. Development of new polymer reagents-stabilizers of drilling fluids//Chemical industry. 2016. Vol. 93 No. 5. Page 262-265.

7. Gaibnazarov S.B. Studying properties of polymer stabilizers of drilling fluids//Chemical industry. 2016. T. 93. No. 4. Page 209-212.

8. Gaibnazarov S.B. The prospects of the using secondary resource in development efficient bore solution //Austrian Journal of Technical and Natural Sciences. Austria, Vienna, 2016. № 3-4. P. 114-117.

\section{AUTHORS PROFILE}

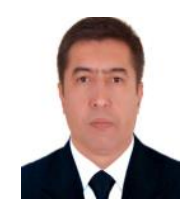

Raupov Anvar Abdirashidovich, Senior Researcher Applicant, Tashkent State Technical University, Tashkent, Uzbekistan Email: raupov.a@gov.uz

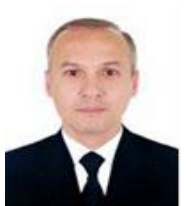

Gaibnazarov Sunnatilla Bahodirjanovich*, Dean of the Department of Mining and Metallurgy, Tashkent State Technical University, Tashkent, Uzbekistan Email: sunnatilla77@mail.ru 